\title{
Dephasing times in quantum dots due to elastic LO phonon-carrier collisions
}

Uskov, A. V.; Jauho, Antti-Pekka; Tromborg, Bjarne; Mørk, Jesper; Lang, R.

Published in:

Physical Review Letters

Link to article, DOI:

10.1103/PhysRevLett.85.1516

Publication date:

2000

Document Version

Publisher's PDF, also known as Version of record

Link back to DTU Orbit

Citation (APA):

Uskov, A. V., Jauho, A-P., Tromborg, B., Mørk, J., \& Lang, R. (2000). Dephasing times in quantum dots due to elastic LO phonon-carrier collisions. Physical Review Letters, 85(7), 1516-1519.

https://doi.org/10.1103/PhysRevLett.85.1516

\section{General rights}

Copyright and moral rights for the publications made accessible in the public portal are retained by the authors and/or other copyright owners and it is a condition of accessing publications that users recognise and abide by the legal requirements associated with these rights.

- Users may download and print one copy of any publication from the public portal for the purpose of private study or research.

- You may not further distribute the material or use it for any profit-making activity or commercial gain

- You may freely distribute the URL identifying the publication in the public portal 


\title{
Dephasing Times in Quantum Dots due to Elastic LO Phonon-Carrier Collisions
}

\author{
A. V. Uskov, ${ }^{1,2,4}$ A.-P. Jauho, ${ }^{3}$ B. Tromborg, ${ }^{2}$ J. Mørk, ${ }^{2}$ and R. Lang ${ }^{4}$ \\ ${ }^{1}$ Lebedev Physical Institute, Leninsky prospekt 53, 117924 Moscow, Russia \\ ${ }^{2}$ Research Center COM, Technical University of Denmark, Building 349, DK-2800 Lyngby, Denmark \\ ${ }^{3}$ Mikroelektronik Centret, Technical University of Denmark, Building 345 East, DK-2800 Lyngby, Denmark \\ ${ }^{4}$ Department of Applied Physics, Tokyo University of Agriculture and Technology, Koganei-shi 184-8588, Japan \\ and CREST, Japan Science and Technology Corporation, Japan
}

(Received 20 December 1999)

\begin{abstract}
Interpretation of experiments on quantum dot (QD) lasers presents a challenge: the phonon bottleneck, which should strongly suppress relaxation and dephasing of the discrete energy states, often seems to be inoperative. We suggest and develop a theory for an intrinsic mechanism for dephasing in QDs: second-order elastic interaction between quantum dot charge carriers and LO phonons. The calculated dephasing times are of the order of $200 \mathrm{fs}$ at room temperature, consistent with experiments. The phonon bottleneck thus does not prevent significant room temperature dephasing.
\end{abstract}

PACS numbers: 73.20.Dx, 63.20.Ls, 78.55.Cr, 85.30.Vw

Semiconductor quantum dots (QDs) may pave the way to optoelectronic devices with vastly superior performance compared to present devices [1]. A detailed understanding of QDs optical properties is therefore of utmost importance, both from a basic science and from a technological point of view. The homogeneous linewidth $\Gamma$ of an optical transition, and the dephasing time, or, equivalently, the polarization relaxation time, $T_{2}=2 / \Gamma$, are basic characteristics for the interaction of light with quantum systems. In lasers, the homogeneous linewidth can strongly affect the laser gain. The dephasing time defines the time scale on which coherent interaction of light with medium takes place [2], and, in particular, it gives the ultimate time scale for realization of coherent control in a quantum system (see, for instance, Ref. [3], and references therein).

In bulk and quantum well (QW) semiconductors the dephasing time is usually taken to be the intraband relaxation time [4]. At low carrier densities, this time is set by LO phonon-carrier scattering, while at higher carrier densities carrier-carrier collisions are also important. The energy spectrum of carriers in QDs is discrete, and in this sense the interaction processes in QDs are qualitatively different from those in QWs or bulk materials. For example, if the LO phonon energy does not coincide with the separation of energy levels in a QD, LO phonon-carrier interaction cannot lead to carrier relaxation between these levels (this is the so-called "phonon bottleneck" [5]). At first sight, then, in the absence of this efficient mechanism much longer relaxation and dephasing times could be expected.

Carrier relaxation is a result of inelastic carrier-phonon and carrier-carrier collisions. In contrast, the dephasing time $T_{2}$ is influenced by both inelastic and elastic collisions. Uskov et al. [6] demonstrated recently that elastic collisions of two-dimensional (2D) carriers with carriers confined in self-assembled QDs can lead to substantial collisional broadening ( $T_{2} \simeq 0.1-1 \mathrm{ps}$ at moderate densities of 2D carriers) of QD spectral lines. In this work we study elastic collisions between QD carriers and LO phonons: these collisions can disturb the phase of the carrier wave functions in QDs without changing the populations of the carrier energy levels, and accordingly QD spectral lines will be broadened. We will show that these processes can lead to dephasing times $T_{2}$ of some hundreds of femtoseconds for typical QDs at room temperature. These values are of the order of the dephasing times in bulk materials and QWs, and are in accordance with recent room temperature measurements [7].

Dephasing and broadening of spectral lines in QDs at low temperatures $(<50 \mathrm{~K})$ are usually attributed to interactions of QD carriers with acoustic phonons [8-10]. In this case, the continuum of acoustic phonons leads to a continuous band of satellites around the zero phonon line (ZPL). This band is considered as homogeneous broadening of the formerly discrete QD spectral line, and the dephasing time is calculated from the width of the band. The present paper considers broadening and dephasing via a polar coupling to LO phonons with fixed frequency. The broadening of the ZPL by LO phonons is interpreted as follows. QD carriers can virtually absorb and then emit (or emit and then absorb) LO phonons. This second-order process does not change the final energy of the carrier, but it does change the phase of the carrier wave function. This change of the phase, in turn, implies dephasing of the dipole for the considered optical transition, and accordingly a broadening of the spectral line [11].

The scattering against LO phonons gives rise to fluctuations $\Delta E(t)$ in the transition energy between the electron and hole QD levels: $E(t)=E^{(0)}+E_{s}+\Delta E(t)$, where $E^{(0)}$ is the unperturbed transition energy, and $E_{s}$ is the energy shift due to the LO phonons. For simplicity, we ignore the Coulombic electron-hole interaction. In a classical treatment the line structure function of the optical transition is given by the Fourier transform of the correlation function $\psi_{f}(\tau)=\overline{f(t+\tau) f^{*}(t)}$ for the oscillator $f(t)$ (here 
the overline indicates an average over fluctuations),

$$
f(t) \propto \exp \left\{-(i / \hbar)\left[\left(E^{(0)}+E_{s}\right) t+\int_{0}^{t} d t_{1} \Delta E\left(t_{1}\right)\right]\right\} .
$$

The correlation function $\psi_{f}(\tau)$ can be expressed as [12]

$$
\begin{aligned}
\psi_{f}(\tau) \propto & \exp \left[-i\left(E^{(0)}+E_{s}\right)(\tau / \hbar)\right] \\
& \times \exp \left[-\frac{1}{2 \hbar^{2}} \int_{0}^{\tau} d t_{1} \int_{0}^{\tau} d t_{2} \overline{\Delta E\left(t_{1}\right) \Delta E\left(t_{2}\right)}\right]
\end{aligned}
$$

In this work we must use the quantum mechanical analog of Eq. (1): the fluctuations in the transition energy are due to interactions with optical phonons, which obey Bose statistics. Thus, instead of $\psi_{f}(\tau)$ we consider [13]

$$
A(\tau)=\left\langle\hat{T} \exp \left\{-(i / \hbar)\left[E^{(0)} \tau+\int_{t}^{t+\tau} d t^{\prime} \hat{H}\left(t^{\prime}\right)\right]\right\}\right\rangle,
$$

where $\hat{T}$ is the time-ordering operator and $\hat{H}(t)$ is the effective interaction Hamiltonian describing the fluctuations in the transition energy. $\langle\cdots\rangle$ indicates an average over the phonon ensemble. The result of our calculations, to be described below, is that the line shape function, which is just the Fourier transform of (3), can be expressed in a compact form:

$$
\begin{aligned}
A(\omega)= & \int d \tau e^{i\left(\hbar \omega-E^{(0)}-E_{s}\right)(\tau / \hbar)} \\
& \times \exp \left\{-\frac{\sigma^{2}}{2 \gamma_{\mathrm{LO}}}\left[|\tau|+\frac{1}{2 \gamma_{\mathrm{LO}}}\left(e^{-2 \gamma_{\mathrm{LO}}|\tau|}-1\right)\right]\right\} .
\end{aligned}
$$

The shift $E_{s}$ and the parameter $\hbar \sigma$, which has the meaning of average quadratic fluctuation of the spectral line, depend on temperature, phonon interaction mechanism, and quantum dot geometry, and explicit expressions for them are given below. $\tau_{\mathrm{LO}}=1 / 2 \gamma_{\mathrm{LO}}$ is the lifetime of optical phonons, which is finite because of various interaction mechanisms (such as phonon-phonon scattering or boundary scattering), and it enters our theory as a parameter which must be calculated separately, or extracted from experiment. If $\sigma \ll 2 \gamma_{\mathrm{LO}}, A(\omega)$ has a Lorentzian line form with FWHM $\Gamma=\sigma^{2} / \gamma_{\mathrm{LO}}$, while, if $\sigma \gg 2 \gamma_{\mathrm{LO}}$, Eq. (4) gives rise to a Gaussian line with width $\Gamma=2 \sqrt{2 \ln 2} \sigma$.

In order to arrive at Eq. (4) the following steps were needed. First, we derive an effective Hamiltonian describing the interaction between quantum dot charge carriers and optical phonons. Next, the time-ordered expectation value (3) must be evaluated. Finally, the developed formalism will be applied to a concrete model of a quantum dot, and the temperature dependence of $\Gamma$ is computed.

The carrier-phonon interaction is $(x=e$ for electrons, $x=h$ for holes)

$$
\hat{U}^{x}(\mathbf{r}, t)=\sum_{\mathbf{q}} C_{q}^{x}\left[a_{\mathbf{q}}(t) e^{i \mathbf{q} \cdot \mathbf{r}}+\text { H.c. }\right],
$$

where $a_{\mathbf{q}}$ is the annihilation operator for the LO phonon with the wave vector q. For polar carrier-phonon coupling $C_{q}^{e}=-C_{q}^{h}$. The finite lifetime of the optical phonons gives rise to the following expression for the phonon Green function [13]:

$$
\begin{aligned}
D(\mathbf{q}, \tau) & \equiv\left\langle\hat{T}\left\{\left[a_{\mathbf{q}}(\tau)+a_{\mathbf{q}}^{\dagger}(\tau)\right]\left[a_{\mathbf{q}}(0)+a_{\mathbf{q}}^{\dagger}(0)\right]\right\}\right\rangle \\
& =e^{-\gamma_{\mathrm{LO}}|\tau|}\left[(\bar{n}+1) e^{-i \omega_{\mathrm{LO}}|\tau|}+\bar{n} e^{i \omega_{\mathrm{LO}}|\tau|}\right],
\end{aligned}
$$

where $\bar{n}=1 /\left[\exp \left(\hbar \omega_{\mathrm{LO}} / k_{B} T\right)-1\right]$, and $\omega_{\mathrm{LO}}$ is the LO phonon frequency. Assuming that the phonon energy $\hbar \omega_{\mathrm{LO}}$ is much less than the energy separation between the QD levels, an effective Hamiltonian $\hat{H}(t)$ can be derived by neglecting real transitions beween the levels [8], and applying perturbation theory in the carrierphonon interaction. Up to second order one finds

$$
\hat{H}^{(1)}(t)=\sum_{\mathbf{q}}\left[f(\mathbf{q}) a_{\mathbf{q}}(t)+f^{*}(\mathbf{q}) a_{\mathbf{q}}^{\dagger}(t)\right],
$$

Here

$$
\hat{H}^{(2)}(t)=-\sum_{\mathbf{q} \mathbf{q}^{\prime}}\left[a_{\mathbf{q}}(t) a_{\mathbf{q}^{\prime}}(t) F\left(\mathbf{q},-\mathbf{q}^{\prime}\right)+a_{\mathbf{q}}(t) a_{\mathbf{q}^{\prime}}^{\dagger}(t) F\left(\mathbf{q}, \mathbf{q}^{\prime}\right)+a_{\mathbf{q}}^{\dagger}(t) a_{\mathbf{q}^{\prime}}(t) F\left(-\mathbf{q},-\mathbf{q}^{\prime}\right)+a_{\mathbf{q}}^{\dagger}(t) a_{\mathbf{q}^{\prime}}^{\dagger}(t) F\left(-\mathbf{q}, \mathbf{q}^{\prime}\right)\right] .
$$

$$
F\left(\mathbf{q}, \mathbf{q}^{\prime}\right)=\left|C_{q} C_{q^{\prime}}\right| \sum_{p>1}\left[\frac{M_{\mathbf{q}}^{e p} M_{\mathbf{q}^{\prime}}^{e p *}}{E_{e p}^{0}-E_{e 1}^{0}}+\frac{M_{\mathbf{q}}^{h p} M_{\mathbf{q}^{\prime}}^{h p *}}{E_{h p}^{0}-E_{h 1}^{0}}\right] .
$$

$E_{e p}^{0}\left(E_{h p}^{0}\right)$ is the unperturbed electron (hole) energy with the wave function $\psi_{e p}\left(\psi_{h p}\right)$, which enters via the matrix element

$$
M_{\mathbf{q}}^{x p}=\int d \mathbf{r} \psi_{x p}^{*}(\mathbf{r}) \exp (i \mathbf{q} \cdot \mathbf{r}) \psi_{x 1}(\mathbf{r})
$$

We consider broadening of the transition between electron and hole ground states $(p=1)$, so that $E^{(0)}=$ $E_{g}+E_{e 1}^{0}+E_{h 1}^{0}$, where $E_{g}$ is the energy gap of the QD material.
The first-order term Eq. (7) is just the standard HuangRhys Hamiltonian [8], while the second-order term (8) is an extension of this theory. A similar second-order term has earlier been shown to lead to broadening of the ZPL in case of impurities in doped crystals [11]. Below we show that the Hamiltonian (7)-(8), which involves only virtual transitions between the QD levels, leads to a broadening of the ZPL in QDs in accordance with experiments [7]. The existence of the nearby levels is essential for obtaining the broadening. The effects of mixing of the QD levels due to transitions between the levels on intensities of phonon satellites have recently been considered in [14].

The evaluation of (3) with the effective Hamiltonian (7) -(8) can be carried out exactly using the cumulant technique [15]. The result is (note that cross terms vanish 
because they involve an unequal number of phonons)

$$
\begin{aligned}
A(\tau)=\exp \{ & -i\left[E^{(0)}+\sum_{j=1,2}\left\langle\hat{H}^{(j)}\right\rangle\right](\tau / \hbar) \\
& -\frac{1}{2 \hbar^{2}} \int_{0}^{\tau} d t_{1} \int_{0}^{\tau} d t_{2} \\
& \left.\times \sum_{j=1,2}\left\langle\hat{T} \Delta \hat{H}^{(j)}\left(t_{1}\right) \Delta \hat{H}^{(j)}\left(t_{2}\right)\right\rangle\right\},
\end{aligned}
$$

where $\Delta \hat{H}^{(j)}(t)=\hat{H}^{(j)}(t)-\left\langle\hat{H}^{(j)}\right\rangle$. It is interesting to note the formal similarity with the classical result Eq. (2). The phonon averages can be carried out with the help of Wick's theorem, which stipulates that the various operator averages implicit in (12) are given as the sum of all pairwise averages. The time-ordered expectation value due to $\hat{H}^{(1)}$ is just $\int_{0}^{t} d t_{1} \int_{0}^{t} d t_{2} \sum_{\mathbf{q}}|f(\mathbf{q})|^{2} D\left(\mathbf{q}, t_{1}-t_{2}\right)$; this expression is well known, say, from studies of phonon-assisted tunneling in resonant-tunneling diodes [16]. In our case this term gives the Huang-Rhys shift $E_{s}^{(1)}=-S \hbar \omega_{\mathrm{LO}}$ of the spectral line, where $S=\sum_{\mathbf{q}}\left|f(\mathbf{q}) /\left(\hbar \omega_{\mathrm{LO}}\right)\right|^{2}$ is the Huang-Rhys parameter $[8,13]$, as well as the satellites at energies $\pm n \hbar \omega_{\mathrm{LO}}$ from the main line. For the present purposes it is important to realize that the first-order term $\hat{H}^{(1)}$ cannot lead to broadening of the ZPL if $\gamma_{\mathrm{LO}} / \omega_{\mathrm{LO}} \ll 1$; see also Ref. [17]. A straightforward calculation shows that the contribution due to $\hat{H}^{(2)}$ is obtained by a replacement $|f(\mathbf{q})|^{2} D\left(t_{1}-t_{2}\right) \rightarrow F\left(\mathbf{q}, \mathbf{q}^{\prime}\right)\left[F\left(\mathbf{q}^{\prime}, \mathbf{q}\right)+F\left(-\mathbf{q},-\mathbf{q}^{\prime}\right)\right] \times$ $D\left(\mathbf{q}, t_{1}-t_{2}\right) D\left(\mathbf{q}^{\prime}, t_{1}-t_{2}\right)$. This expression generates two kinds of terms: (i) Terms proportional to $\exp \left[ \pm 2 i \omega_{\mathrm{LO}}\left(t_{1}-t_{2}\right)\right]$, together with $\left\langle\hat{H}^{(2)}\right\rangle$ in (12), give the spectral shift in the second-order theory. They are also relevant for the two-phonon satellites at $\pm n 2 \hbar \omega_{\mathrm{LO}}$ of the main line, which however is not our focus here. (ii) Terms without these fast time oscillations which determine the zero phonon linewidth. Collecting all the terms describing the spectral shift and broadening, we find

$$
\begin{gathered}
E_{s}^{(2)}=-(2 \bar{n}+1)\left(S_{1}+S_{2}\right) \hbar \omega_{\mathrm{LO}}, \\
\sigma=\sqrt{4 \bar{n}(\bar{n}+1) S_{2}} \omega_{\mathrm{LO}},
\end{gathered}
$$

where

$$
\begin{gathered}
S_{1}=\sum_{\mathbf{q}} F(\mathbf{q}, \mathbf{q}) /\left(\hbar \omega_{\mathrm{LO}}\right) \\
S_{2}=\sum_{\mathbf{q} \mathbf{q}^{\prime}} F\left(\mathbf{q}, \mathbf{q}^{\prime}\right)\left[F\left(\mathbf{q}^{\prime}, \mathbf{q}\right)+F\left(-\mathbf{q},-\mathbf{q}^{\prime}\right)\right] / 2\left(\hbar \omega_{\mathrm{LO}}\right)^{2}
\end{gathered}
$$

The second-order shift (13) together with the first-order shift $E_{s}^{(1)}$ gives the total shift $E_{s}$ of the ZPL in (4). The parameters $S_{1}$ and $S_{2}$ are extensions of the parameter $S$ of the Huang-Rhys theory. Our numerical calculations show that $S_{1}, S_{2} \ll 1$, and also $S_{1} \gg S_{2}$. Equation (14) together with (4) allows an analysis of the spectral line shape, and, in particular, its width as a function of temperature.
As a practical application, we consider a cylindrical quantum dot of radius $R$ and height $H$, with infinite confining potential. In the calculation of the average fluctuation $\sigma$, we include in Eq. (10) only the leading terms in the sum over the states $p$. If $2 R>H$, two states have the same energy, and are the closest to the ground state. Taking into account this twofold degeneracy, we get

$$
\hbar \sigma=\sqrt{\bar{n}(\bar{n}+1)} \frac{\hbar \omega_{\mathrm{LO}}}{\Delta \tilde{E}} g(H / R) \frac{K}{R},
$$

where $\Delta \tilde{E}=\hbar^{2} /\left[2\left(m_{e}+m_{h}\right) R^{2}\right]\left[\alpha_{11}^{2}-\alpha_{10}^{2}\right] . \quad \alpha_{l m}$ is the $l$ th root of the Bessel function of the order $m$. The material parameter $K=e^{2}\left(1 / \epsilon_{\infty}-1 / \epsilon\right) /\left(\pi^{2} \epsilon_{0}\right)$ has the value $26.0 \mathrm{meV} \mathrm{nm}$ for GaAs. The dimensionless function $g(H / R)$ is shown in the inset of Fig. 1 .

In the numerical calculation of the width $\Gamma$ (see Fig. 1) we use $\tau_{\mathrm{LO}}=1.5 \mathrm{ps}$ [18] so that $\hbar \gamma_{\mathrm{LO}}=0.22 \mathrm{meV}$ and $\left(\omega_{\mathrm{LO}} \tau_{\mathrm{LO}}\right)^{-1} \simeq 0.01$. From (17) one finds that at $T=$ $300 \mathrm{~K}$ the average quadratic fluctuation is $\hbar \sigma \simeq 2.2 \mathrm{meV}$ [corresponding to $S_{2} \simeq 2 \times 10^{-3}$ in Eq. (14)], so that the condition $\sigma \gg 2 \gamma_{\mathrm{LO}}$ is satisfied, and consequently the spectral line has a Gaussian shape. At lower temperatures $\sigma$ decreases and can reach the regime $\sigma \ll 2 \gamma_{\mathrm{LO}}$, where the Lorentzian shape obtains. At $T=300 \mathrm{~K}$ the linewidth $\hbar \Gamma$ is $5.1 \mathrm{meV}$, corresponding to $T_{2} \simeq 250 \mathrm{fs}$. Taking into account higher excited states in the sum (10) in evaluating $\sigma$ typically reduces $T_{2}$ by a factor of 1.5-2. Thus, one can expect room temperature dephasing times of the order of 100-200 fs. This is in good agreement with experimental values (200-300 fs) [7]. The LO phonon contribution to the linewidth is proportional to $\bar{n}(\bar{n}+1)$ if $\Gamma \ll 1 / \tau_{\mathrm{LO}}$, and thus vanishes as the temperature tends to zero. For example, for $T<50 \mathrm{~K}$ the calculated values of $\hbar \Gamma$ are below $20 \mu \mathrm{eV}$ (see Fig. 1), which is below the linewidths found in experiment $(\simeq 50-150 \mu \mathrm{eV})[9,10]$. On the other hand, the acoustic phonon related linewidth does not vanish in this limit [9], and we conclude that the

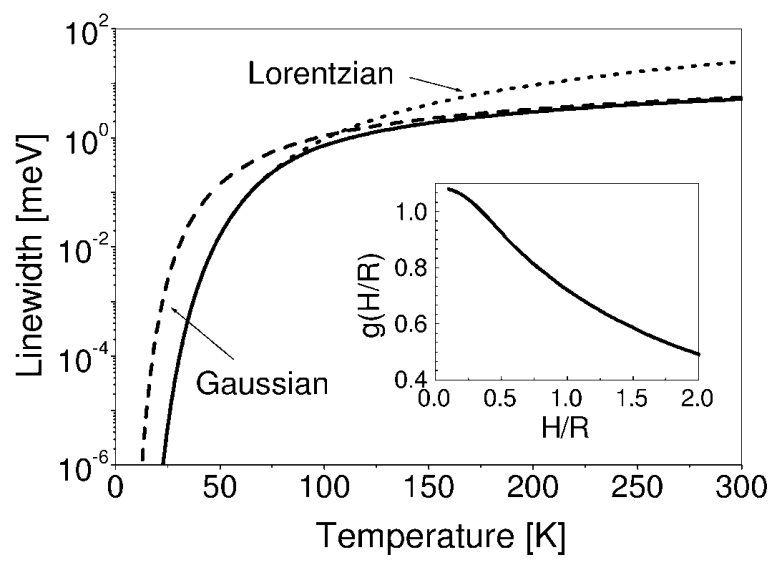

FIG. 1. The linewidth as a function of temperature. The following parameters were used: $\epsilon_{\infty}=10.9, \epsilon=12.9, m_{e}+$ $m_{h}=0.35 m_{0}$, and $R=H=5 \mathrm{~nm}$. The solid curve is the result of a full numerical calculation, while the Lorentzian and Gaussian approximations are given as dotted and dashed lines, respectively. The inset shows the function $g(R / H)$. 
LO mechanism is dominant only at elevated temperatures, say $T>100 \mathrm{~K}$. The present broadening mechanism also provides a possible way of understanding fast carrier relaxation in QDs at elevated temperatures [19]: because of the substantial broading of the levels their differences no longer have to match strictly the LO phonon energy, thereby weakening the phonon bottleneck effect.

It is also instructive to consider the classical limit of our calculations. Then $\bar{n} \gg 1$, and thus for $\Gamma \ll 1 / \tau_{\text {LO }}$ we find $\Gamma \propto \bar{n}^{2}$. This result also follows from Eq. (2) if we treat the phonons as classical random fields perturbing the QD carriers. This classical result allows one to establish an analogy with broadening in atomic gases [20]. There, elastic atomic collisions lead to dephasing of optical transitions, and the homogeneous linewidth in the Lorentzian limit is proportional to the density of the colliding particles. In our case the broadening of ZPL is the result of elastic second-order interactions between phonons and the carriers, and hence $\Gamma \propto \bar{n}^{2}$. Note also that the LO phonon lifetime $\tau_{\text {LO }}$ plays the same role as the collisional time in atomic gases [20].

We finally consider the effect of the shape and size of the QD on the dephasing time. Equation (17) implies that $\sigma \propto$ $L$, where $L$ characterizes the size of the QD. In the Lorentz case $\Gamma \propto \sigma^{2} \propto L^{2}$, while in the Gaussian case $\Gamma \propto \sigma \propto$ $L$. From Fig. 1 we can see that $\Gamma$ can be modified by a factor of 2-3 by changing the ratio $H / R$ of the cylinder. Note also that $\sigma$ and $\Gamma$ depend on the carrier masses, $\sigma \propto\left(m_{e}+m_{h}\right)$. These characteristic dependencies point towards a possibility to engineer the spectral linewidths.

In conclusion, we have developed a theory for dephasing of an optical transition in QDs due to second-order elastic interaction with LO phonons. The theory results in expressions which are easily evaluated numerically, and can also be used to draw qualitative conclusions about the dependence of the dephasing time on physical parameters defining the QD. At room temperature, despite an apparent phonon bottleneck, the considered mechanism leads to dephasing times of the order of $200 \mathrm{fs}$, i.e., of the same order as in bulk, and also as observed in QDs.

One of us (A. V.U.) thanks the Ministry of Education, Science, Culture and Sport of Japan for support during his work at TUAT. The work of A. V. U. was also supported by the Russian Federation Program "Integration" (Project No. A0155). We acknowledge D. Birkedal, P. Borri, and J. Hvam for useful comments.

[1] D. Bimberg, M. Grundmann, and N. N. Ledentsov, Quantum Dot Heterostructures (Wiley \& Sons, New York, 1999).
[2] P. Meystre and M. Sargent III, Elements of Quantum Optics (Springer-Verlag, Berlin, 1990).

[3] M. N. Bonadeo, J. Erland, D. Gammon, D. Park, D. S. Katzer, and D. G. Steel, Science 282, 1473 (1998).

[4] W. W. Chow, S. W. Koch, and M. Sargent III, Semiconductor-Laser Physics (Springer-Verlag, Berlin, 1994).

[5] H. Benisty, C. M. Sotomayor-Torres, and C. Weisbuch, Phys. Rev. B 44, 10945 (1991).

[6] A. V. Uskov, K. Nishi, and R. Lang, Appl. Phys. Lett. 74, 3081 (1999).

[7] P. Borri, W. Langbein, J. Mørk, J. M. Hvam, F. Heinrichsdorff, M.-H. Mao, and D. Bimberg, Phys. Rev. B 60, 7784 (1999).

[8] S. Schmitt-Rink, D. A. B. Miller, and D. S. Chemla, Phys. Rev. B 35, 8113 (1987).

[9] T. Takagahara, Phys. Rev. B 60, 2638 (1999).

[10] K. Ota, N. Usami, and Y. Shiraki, Physica (Amsterdam) 2E, 573 (1998).

[11] A similar approach has earlier been used for the description of optical dephasing in doped crystals, see, e.g., M. A. Krivoglaz, Fiz. Tverd. Tela 6, 1701 (1964), or I. S. Osad'ko, Phys. Rep. 206, 45 (1991).

[12] See, for example, Sect. 6.5.3 in G. P. Agrawal and N. K. Dutta, Semiconductor Lasers (Van Nostrand Reinhold, New York, 1993), 2nd ed.

[13] G. D. Mahan, Many-Particle Physics (Plenum Press, New York, 1990), 2nd ed.

[14] V. M. Fomin, V. N. Gladilin, J. T. Devreese, E. P. Pokatilov, S. N. Balaban, and S. N. Klimin, Phys. Rev. B 57, 2415 (1998).

[15] A discussion of models which can be solved exactly with the linked cluster expansion can be found in Sect. 4.3.F of Ref. [13]. Obviously, the full electron-phonon interaction (5) does not belong to this class.

[16] N. S. Wingreen, K. W. Jacobsen, and J. W. Wilkins, Phys. Rev. B 40, 11834 (1989).

[17] We also note that the amplitude $f(\mathbf{q})$ is small, because the electron and hole contributions tend to cancel for polar coupling. In particular, $f(\mathbf{q}) \equiv 0$, if the electron and hole wave functions are equal, such as in an infinitely deep well.

[18] Both theory, e.g., B. K. Ridley and R. Gupta, Phys. Rev. B 43, 4939 (1991) or X.-Q. Li and Y. Arakawa, Phys. Rev. B 57, 12285 (1998), and experiments, J. A. Kash, J. C. Tsang, and J. M. Hvam, Phys. Rev. Lett. 54, 2151 (1985) suggest $\tau_{\mathrm{LO}}$ of the order of $2-4 \mathrm{ps}$ at room temperature. It is important to note that at room temperature our theory yields linewidths which are essentially independent of $\tau_{\mathrm{LO}}$.

[19] For recent experiments, see, e.g., T. S. Sosnowski, T. B. Norris, H. Jiang, J. Singh, K. Kamath, and P. Bhattacharya, Phys. Rev. B 57, R9423 (1998), or S. Marcinkevičius and R. Leon, Phys. Rev. B 59, 4630 (1999).

[20] W. Demtroeder, Laser Spectroscopy (Springer-Verlag, Berlin, 1996). 